\title{
Hipertensión arterial pulmonar por ecocardiografía en pacientes con enfermedades de tejido conectivo
}

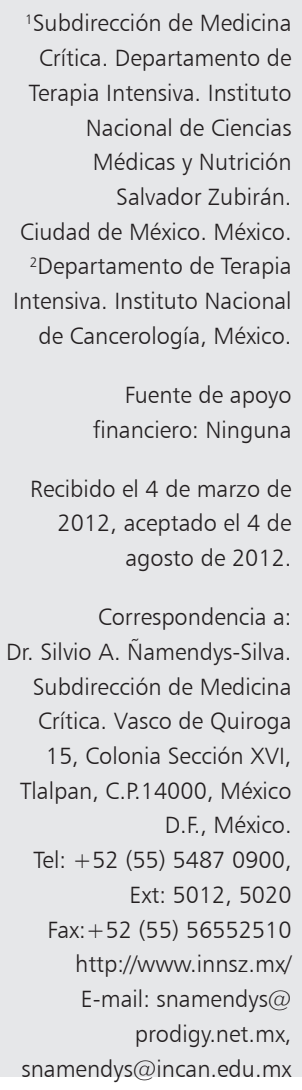

Subdirección de Medicina Crítica. Departamento de Terapia Intensiva. Instituto Nacional de Ciencias Médicas y Nutrición Salvador Zubirán. Ciudad de México. México. ${ }^{2}$ Departamento de Terapia Intensiva. Instituto Nacional de Cancerología, México.

Fuente de apoyo financiero: Ninguna

Recibido el 4 de marzo de 2012, aceptado el 4 de agosto de 2012

Correspondencia a: Dr. Silvio A. Ñamendys-Silva. Subdirección de Medicina Crítica. Vasco de Quiroga 15, Colonia Sección XVI, Tlalpan, C.P.14000, México D.F., México.

Tel: +52 (55) 54870900 , Ext: 5012,5020

Fax:+52 (55) 56552510 http://www.innsz.mx/ E-mail: snamendys@ prodigy.net.mx,

snamendys@incan.edu.mx

\author{
SILVIO ANTONIO ÑAMENDYS-SILVA ${ }^{1,2}$, \\ MARÍA OCOTLÁN GONZÁLEZ-HERRERA ${ }^{1,2}$, \\ JOSÉ LUIS HERNÁNDEZ-OROPEZA ${ }^{1}$, \\ EDUARDO RIVERO-SIGARROA ${ }^{1}$, GUILLERMO DOMÍNGUEZ-CHERIT ${ }^{1}$
}

Background: Pulmonary arterial hypertension is an important cause of complications among patients with connective tissue diseases. Aim: To describe the clinical and echocardiographic characteristics of patients with pulmonary hypertension associated with connective tissue diseases. Material and Methods: Retrospective, observational and descriptive study. We analyzed 35 patients with pulmonary hypertension associated with connective tissue diseases. All patients were evaluated and diagnosed by at least one medical specialist in rheumatology. Pulmonary arterial hypertension was defined as a pulmonary artery systolic pressure $\geq 40 \mathrm{mmHg}$ by echocardiography. The group was divided as not severe when pressures ranged from 40 to $64 \mathrm{mmHg}$ and severe, when pressures were $\geq 65 \mathrm{mmHg}$. Results: The most common connective tissue disease associated with pulmonary arterial hypertension was diffuse scleroderma in $46 \%$ of cases. Eighty nine percent of patients were female. Time of evolution of the pulmonary hypertension was $18.8 \pm 21.8$ months. The distance walked in the six minute walk test was $<400 \mathrm{~m}$ both in patients with and without severe pulmonary hypertension. Fifty one percent of patients had pulmonary restriction. No differences in gas exchange parameters were observed between groups. Comparing echocardiographic findings in patients with and without severe hypertension, the former had a higher frequency of right ventricular dilatation (85.7 and 52.3\% respectively, $p$ $=0.04)$, right ventricular hypertrophy (42.8 and $0 \%$ respectively, $p=0.02)$ and right ventricular hypokinesia ( 71.4 and $9.5 \%$ respectively $p=<0.01$ ). Conclusions: Patients with severe pulmonary arterial hypertension associated to connective tissue diseases have more commonly dilated, hypertrophic and hypokinetic right ventricles.

(Rev Med Chile 2013; 141: 58-62).

Key words: Connective tissue diseases; Echocardiography; Pulmonary.

L a hipertensión arterial pulmonar (HAP) es una de las principales causas de morbilidad $y$ mortalidad en pacientes con enfermedades de tejido conectivo (ETC). La HAP asociada a ETC pertenece al grupo 1 de acuerdo a la clasificación clínica de Hipertensión Pulmonar Dana Point, $2008^{1}$. Los mecanismos fisiopatológicos impli- cados son la disfunción endotelial y remodelado vascular; como resultado del desequilibrio en la sobreexpresión de sustancias vasoconstrictoras, trombogénicas, mitogénicas y reducción de vasodilatadores $^{2}$. La HAP se define por cateterismo cardiaco como la presión media de arteria pulmonar (PAPm) mayor de $25 \mathrm{mmHg}$ en reposo, con pre- 
sión capilar pulmonar $<15 \mathrm{mmHg}$ y resistencias pulmonares $\geq 2$ ó 3 unidades Wood ${ }^{1}$. Por ecocardiograma se considera HAP a partir de una presión sistólica de arteria pulmonar (PSAP) $>40 \mathrm{mmHg}$; y se clasifica de acuerdo a esta en: leve de 40 a $54 \mathrm{mmHg}$, moderada de 55 a $64 \mathrm{mmHg}$ y grave cuando es $>65 \mathrm{mmHg}^{3}$. El ecocardiograma transtorácico bidimensional y el ecocardiograma (ECG) doppler se ha convertido en una herramienta clave para la detección de HAP en pacientes con $\mathrm{ETC}^{4}$. El ECG Doppler transtorácico como método no invasivo permite el diagnóstico de HAP en pacientes asintomáticos, se sugiere realizar anualmente en pacientes portadores de escleroderma y en quienes presentan síntomas relacionados con HAP en otras patologías del tejido conectivo ${ }^{5}$. Este estudio de detección temprana de HAP tiene sensibilidad del $90 \%$ y especificidad del $75 \%{ }^{6}$. Además de tener utilidad para excluir hipertensión pulmonar asociada a cardiopatías (grupo 2). Se han descrito predictores ecocardiográficos de mal pronóstico en pacientes con HAP como derrame pericárdico recurrente, dilatación auricular y ventricular derecha, índice de esfericidad del ventrículo izquierdo (Lei) $>1,2$, índice de rendimiento miocárdico del ventrículo derecho (Tei) $>1,4$, dilatación de la vena cava inferior y excursión sistólica del anillo tricuspídeo (TAPSE) $<1,8 \mathrm{~cm}^{7}$. La prevalencia de HAP en escleroderma es del 13,3\%, siendo menor en otras patologías como enfermedad mixta de tejido conectivo, lupus eritematoso generalizado, artritis reumatoide, dermatomiositis, polimiositis y síndrome Sjögren ${ }^{8}$. La prevalencia de HAP en pacientes con ETC en México no se conoce. El propósito del presente estudio fue describir las características clínicas y ecocardiográficas de los pacientes con HAP asociada a ETC

\section{Material y Métodos}

Estudio observacional realizado de marzo de 2008 a julio de 2009 en el Instituto Nacional de Ciencias Médicas y Nutrición Salvador Zubirán (INCMNSZ). Se incluyeron pacientes de ambos géneros, mayores de 16 años, atendidos en la clínica de hipertensión pulmonar del INCMNSZ con diagnóstico de HAP asociada a ETC. En todos los casos, la ETC fue diagnosticada por un médico especialista en reumatología. Los datos se obtuvieron a través de la revisión de los expedientes clínicos.
Los pacientes con expediente clínico incompleto fueron excluidos del estudio. Se utilizó estadística descriptiva para la presentación de los datos. Se realizó la prueba de Kolmogorov-Smirnov para determinar la distribución de los datos. Las variables numéricas se expresan como media \pm desviación estándar o como mediana y rangos intercuartilares para las variables con distribución no paramétrica y las variables nominales en porcentaje. Para comparar las variables continuas se utilizo prueba to U de Mann-Whitney de acuerdo a la distribución muestral. Para el análisis de variables categóricas se utilizó la prueba de $\chi^{2}$ o prueba exacta de Fisher de dos colas según correspondiera. En todos los casos, un valor de $\mathrm{p}<0,05$ fue considerado como estadísticamente significativo. Se utilizó el paquete estadístico SPSS 16.0 para realizar la estadística descriptiva e inferencial. Se definió hipertensión arterial pulmonar si la presión sistólica arterial pulmonar fue $\geq 40 \mathrm{mmHg}$ por ecocardiografía, el grupo de dividió en no grave $(40-64 \mathrm{mmHg}$ ) y grave $\geq 65 \mathrm{mmHg}^{3}$. La revisión de los expedientes clínicos de los pacientes incluidos en el estudio fue aprobada por la Subdirección de Medicina Crítica del INNCMSZ. Por tratarse de un estudio retrospectivo no se requirió consentimiento informado.

\section{Resultados}

Se analizaron 137 pacientes con hipertensión pulmonar, 35 pacientes (25\%) presentaron HAP asociada a ETC, siendo más frecuente en pacientes con escleroderma difusa (45,7\%) (Figura 1). El género más frecuentemente afectado fue el femenino $(88,6 \%)$. La media de edad fue de 45,97 \pm 13,2 años; el tiempo de evolución de HAP fue de $18,80 \pm 21,8$ meses; y el tiempo de evolución de la enfermedad del tejido conectivo fue 10,23 $\pm 11,7$ años. La presión sistólica de la arteria pulmonar medida por ecocardiografía doppler fue de 68,37 $\pm 26,2 \mathrm{mmHg}$ (Tabla 1). La distancia recorrida evaluada por caminata de 6 min en ambos grupos con y sin HAP grave fue menor a 400 metros $(\mathrm{p}=$ 0,986 ) (Tabla 2). No hubo diferencia entre ambos grupos para capacidad vital forzada (CVF) predominando como patrón espirométrico la restricción pulmonar (Figura 2); no hubo diferencias en la presión arterial de oxígeno $\left(\mathrm{PaO}_{2}\right)$, presión arterial $\mathrm{CO}_{2}\left(\mathrm{PaCO}_{2}\right)$ y saturación arterial $\mathrm{O}_{2}\left(\mathrm{SaO}_{2}\right)$ (Tabla 3). Al comparar los grupos sin y con HAP grave 
Hipertensión arterial pulmonar asociada a enfermedades de tejido conectivo - S. A. Ñamendys-Silva et al

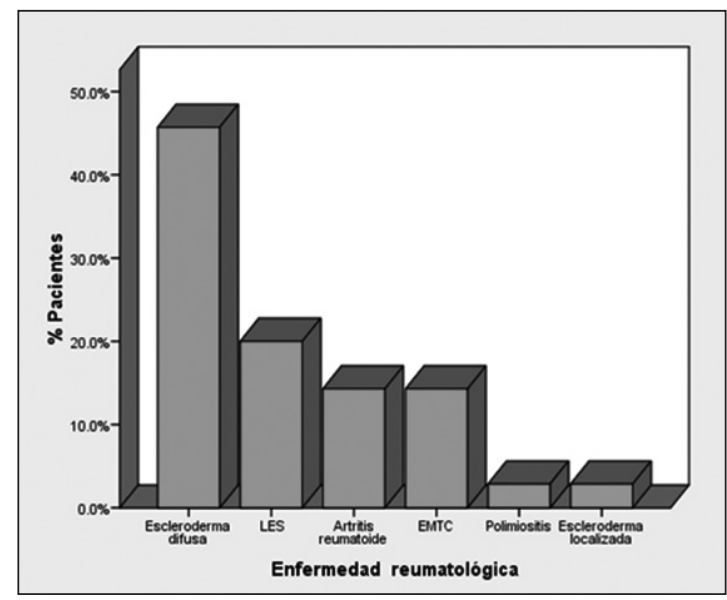

Figura 1. Distribución de enfermedades de tejido conectivo en pacientes con hipertensión arterial pulmonar. $\mathrm{EMTC}=$ enfermedad mixta de tejido conectivo, LEG = lupus eritematoso generalizado.

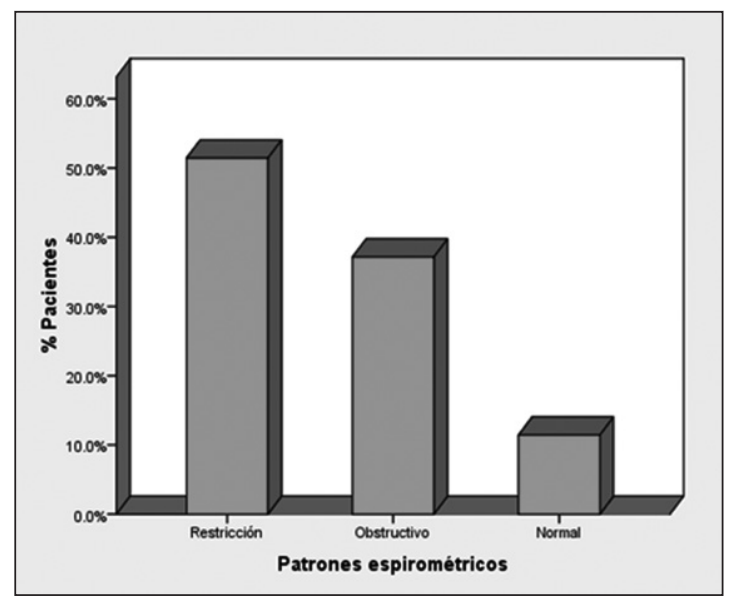

Figura 2. Patrones espirométricos en enfermos con hipertensión arterial pulmonar asociada a enfermedades de tejido conectivo.

\section{Tabla 1.Características demográficas} de pacientes con HAP asociada a ETC

\begin{tabular}{|lc|}
\hline Variables & $\mathbf{n}=\mathbf{3 5}$ \\
\hline Femenino (\%) & $31(88,6)$ \\
\hline Edad (años) & $45,97 \pm 13,2$ \\
\hline Tiempo de HAP (meses) & $18,80 \pm 21,8$ \\
\hline Tiempo de ETC (años) & $10,23 \pm 11,7$ \\
\hline PSAP (mmHg) & $68,37 \pm 26,2$ \\
\hline Caminata 6 minutos (metros) & $329(329-331)$ \\
\hline
\end{tabular}

$\mathrm{ETC}=$ enfermedad de tejido conectivo, $\mathrm{HAP}=$ hipertensión arterial pulmonar, PSAP=presión sistólica de arteria pulmonar.
Tabla 2. Características clínicas de pacientes con y sin HAP grave

\begin{tabular}{|lccc|}
\hline Variables & $\begin{array}{c}\text { PSAP } \\
<\mathbf{6 5} \mathbf{~ m m H g} \\
(\mathbf{n}=\mathbf{2 1})\end{array}$ & $\begin{array}{c}\text { PSAP } \\
\mathbf{6 5} \mathbf{~ m m H g} \\
\mathbf{( n = 1 4 )}\end{array}$ & p \\
\hline Edad (años) & $44,95 \pm 15,1$ & $47,50 \pm 9,9$ & 0,585 \\
\hline Femenino (\%) & $19(90,4)$ & $12(85,7)$ & 0,530 \\
\hline Tiempo HAP (meses) & $16,71 \pm 16,2$ & $21,93 \pm 28,7$ & 0,498 \\
\hline Tiempo ETC (años) & $11,05 \pm 13,4$ & $9,0 \pm 9,0$ & 0,622 \\
\hline Distancia (metros) & $329(329-331)$ & $329(329-330)$ & 0,986 \\
\hline
\end{tabular}

$\mathrm{ETC}=$ enfermedad de tejido conectivo, $\mathrm{HAP}=$ hipertensión arterial pulmonar.

Tabla 3. Gasometría arterial y pruebas de función respiratoria en pacientes con y sin HAP grave

\begin{tabular}{|lccc|}
\hline Variables & $\begin{array}{c}\text { PSAP }<\mathbf{6 5} \mathbf{~ m m H g} \\
(\mathbf{n}=\mathbf{2 1})\end{array}$ & $\begin{array}{c}\text { PSAP } \mathbf{2} \mathbf{6 5} \mathbf{~ m m H g} \\
(\mathbf{n}=\mathbf{1 4})\end{array}$ & p \\
\hline $\mathrm{PaO}_{2}(\mathrm{mmHg})$ & $63,45 \pm 14,1$ & $56,82 \pm 16,6$ & 0,216 \\
$\mathrm{SaO}_{2}(\%)$ & $90,72 \pm 6,2$ & $86,38 \pm 7,6$ & 0,075 \\
\hline $\mathrm{PaCO}_{2}(\mathrm{mmHg})$ & $34,83 \pm 5,6$ & $35,45 \pm 7,8$ & 0,786 \\
$\mathrm{pH}$ & $7,42 \pm 0,03$ & $7,40 \pm 0,04$ & 0,095 \\
\hline $\mathrm{CVF}(\%$ predicho) & $73,52 \pm 24,54$ & $63,86 \pm 16,21$ & 0,205 \\
\hline $\mathrm{FEV}$ & (\% predicho) & $69,52 \pm 24,55$ & 0,305 \\
\hline $\mathrm{FEV} / \mathrm{CVF}$ & $78,76 \pm 7,48$ & $81,50 \pm 9,26$ & 0,342 \\
\hline
\end{tabular}

$\mathrm{FEV}_{1}=$ volumen espiratorio forzado del primer segundo, CVF= capacidad vital forzada, $\mathrm{PaO}_{2}=$ presión arterial de oxígeno, $\mathrm{PCO}_{2}=$ presión arterial de dióxido de carbono, $\mathrm{SaO}_{2}=$ saturación arterial de oxígeno. 
Tabla 4. Datos ecocardiográficos de pacientes con y sin HAP grave

\begin{tabular}{|c|c|c|c|}
\hline Variables & $\begin{array}{c}\text { PSAP }<65 \mathrm{mmHg} \\
(\mathrm{n}=21)\end{array}$ & $\begin{array}{c}\text { PSAP } \geq 65 \mathrm{mmHg} \\
(\mathrm{n}=14)\end{array}$ & $\mathbf{p}$ \\
\hline FEVI (\%) & $63 \pm 5,4$ & $65,36 \pm 7,3$ & 0,284 \\
\hline Dilatación VD (\%) & $11(52,3)$ & $12(85,7)$ & 0,042 \\
\hline Hipertrofia VD (\%) & $0(0)$ & $6(42,8)$ & 0,002 \\
\hline Hipocinesia VD (\%) & $2(9,5)$ & $10(71,4)$ & $<0,0001$ \\
\hline Dilatación AD (\%) & $14(66,6)$ & $12(85,7)$ & 0,262 \\
\hline Derrame pericárdico (\%) & $4(19)$ & $4(28,5)$ & 0,685 \\
\hline
\end{tabular}

$\mathrm{AD}=$ aurícula derecha, $\mathrm{FEVI}=$ fracción de eyección del ventrículo izquierdo, $\mathrm{VD}=$ ventrículo derecho.

se encontró diferencias en las siguientes variables ecocardiográficas: dilatación del ventrículo derecho $(52,3 \%$ vs $85,7 \% ; p=0,042)$, hipertrofia del ventrículo derecho ( $0 \%$ vs $42,8 \% ; \mathrm{p}=0,002)$ e hipocinesia del ventrículo derecho $(9,5 \%$ vs $71,4 \%$; $\mathrm{p}=<0,0001)($ Tabla 4$)$.

\section{Discusión}

En este estudio encontramos como principales hallazgos que la presencia de dilatación, hipertrofia e hipocinesia del VD están asociados a HAP grave. Mukerjee et $\mathrm{al}^{9}$, reportaron una relación mujer: hombre 4:1. En nuestro estudio el género predominantemente afectado fue el femenino con una proporción $7: 1$, lo que puede estar relacionado con la mayor prevalencia de enfermedades reumatologías en el género femenino. La mayor frecuencia encontrada de HAP asociada a ETC correspondió a escleroderma difusa y en menor frecuencia para otras enfermedades reumatológicas. En relación a la edad de los enfermos la media fue de 45 años, siendo menor a la reportada por otros autores; así como el tiempo de presentación de la HAP como complicación de ETC siendo la media de evolución en nuestro grupo de enfermos de 10 años. La capacidad de ejercicio evaluada por la distancia recorrida en la prueba de caminata de 6 min fue menor a 400 metros para ambos grupos, con y sin HAP grave, a pesar de la gravedad de la HAP no hubo diferencia en distancia recorrida para ambos grupos. Villalba et $\mathrm{al}^{10}$, describieron a la caminata de 6 min menor a 400 metros como factor independiente de mortalidad en pacientes con HAP asociada a escleroderma. El 51\% de en- fermos en nuestro estudio presentaron restricción pulmonar con CVF disminuida en ambos grupos, a pesar de ello no se encontró deterioro en el intercambio gaseoso, una probable explicación a éste hallazgo es la posible presencia de enfermedad pulmonar intersticial descrita en pacientes con HAP asociada a ETC $(18,1 \%)^{11}$. El pronóstico es similar en HAP asociada a ETC con y sin enfermedad intersticial ${ }^{11,12}$. La importancia en la detección de HAP en estadios tempranos o subclínicos en pacientes con ETC mediante ecocardiografía doppler transtorácico ha sido recomendado como método inicial no invasivo para la evaluación de la HAP y su repercusión en la función y tamaño de las cavidades cardiacas derechas, así como para determinar la presencia de derrame pericárdico, los cuales han demostrado estar relacionados con mejor supervivencia en estos enfermos ${ }^{3,13}$. Aunque en el estudio de Fisher et $\mathrm{al}^{3}$, describieron que la estimación por ecocardiografía doppler de la PSAP puede ser frecuentemente subestimada y en menor frecuencia sobrestimada explicado por la inexactitud de la estimación de la presión auricular derecha y del jet de regurgitación trans-tricuspideo; no considerando al ECG Doppler un método de detección exacta para detectar HAP en pacientes asintomáticos o con HAP leve; se continua considerando al ecocardiograma como una herramienta útil para evaluación, manejo y pronóstico de la hipertensión pulmonar a través de parámetros que evalúan la función del ventrículo derecho, determinante fundamental de la evolución de la enfermedad ${ }^{1,3}$. En este estudio se identificaron la dilatación, hipertrofia e hipocinesia ventricular derecha como las variables ecocardiográficas asociadas con gravedad y mal pronóstico de pacientes 
Hipertensión arterial pulmonar asociada a enfermedades de tejido conectivo - S. A. Ñamendys-Silva et al

con HAP grave asociada a ETC, siendo útil para identificar a enfermos que requieren manejo intensivo que mejore su clase funcional.

\section{Limitaciones del estudio}

1. El tamaño de la muestra.

2. Se reporta la experiencia de un solo centro.

3. No se realizó medición directa de las presiones de la circulación pulmonar a través de catéter de flotación en la arteria pulmonar y,

4. No se realizó medición de la capacidad de difusión de monóxido de carbono.

\section{Conclusión}

El 25\% de pacientes con hipertensión arterial presentaron HAP diagnosticada por ecocardiografía asociada a ETC, siendo más frecuente en pacientes del género femenino con escleroderma difusa. Los enfermos con HAP grave presentaron mayor dilatación, hipertrofia e hipocinesia del ventrículo derecho.

\section{Referencias}

1. Nazzareno G, Hoeper M, Humbert M, Torbicki A, Vachiery J, Barbera J, et al. Guidelines for the diagnosis and treatment of pulmonary hypertension. Eur Heart J 2009; 30 (20): 2493-537.

2. Farber H, Loscalzo J. Pulmonary Arterial Hypertension. N Engl J Med 2004; 351 (16): 1655-65.

3. Fisher M, Forfia P, Chamera E, Housten-Harris T, Champion H, Girgis R, et al. Accuracy of Doppler Echocardiography in the Hemodynamic Assessment of Pulmonary Hypertension. Am J Respir Crit Care Med 2009; 179 (7): 615-21.

4. Bossone E, Bodini B, Mazza A, Allegra L. Pulmonary Arterial Hypertension. The Key Role of Echocardiography. Chest 2005; 127 (5): 1836-43.
5. Galiè N, Manes A, Farahani KV, Pelino F, Palazzini M, Negro L, et al. Pulmonary arterial hypertension associated to connective tissue diseases. Lupus 2005; 14 (9): 713-7.

6. Bull T. Screening and therapy of pulmonary hypertension in systemic sclerosis. Curr Opin Rheumatol 2007; 19 (6): 598-603.

7. Mereles D, Grünig E. A Stepwise and Practical Approach to Optimizing Echocardiography in Pulmonary Hypertension. Advances in Pulmonary Hypertension Autumn 2006; 5 (3) 30-3.

8. Wigley FM, Lima JA, Mayes M, McLain D, Chapin JL, Ward-Able C. The prevalence of undiagnosed pulmonary arterial hypertension in subjects with connective tissue disease at the secondary health care level of community-based rheumatologists (the UNCOVER study). Arthritis Rheum 2005; 52 (7): 212532.

9. Mukerjee D, St George S, Coleiro B, Kninght C, Denton C, Davar J, et al. Prevalence and outcome in systemic sclerosis associated pulmonary arterial hypertension: application of a registry approach. Ann Rheum dis 2003; 62 (11): 1088-93.

10. Villalba W, Sampaio-Barros P, Pereira M, Cerqueira E, Leme C, Marques-Neto J, et al. Six minute walk test for the evaluation of pulmonary disease severity in scleroderma patients. Chest 2007; 131 (1): 217-22.

11. Chang B, Wigley FM, White B, Wise RA. Scleroderma patients with combined pulmonary hypertension and interstitial lung disease. J Rheumatol 2003; 30 (11): 2398-405.

12. Ghofrani HA, Voswinckel R, Reichenberger F, Weissmann N, Schermuly RT, Seeger W, et al. Hypoxia and non-hypoxia- related pulmonary hypertensionEstablished and new therapies. Cardiovascular Research 2006; 72 (1): 30-40.

13. Barst RJ, McGoon M, Torbicki A, Sitbon O, Krowka MJ, Olschewski H, et al. Diagnosis and differential assessment of pulmonary arterial hypertension. J Am Coll Cardiol 2004; 43 (12 Suppl S): 40S-7S. 